\title{
Mapping from Statistical to Biological Proximity
}

\author{
Malay Bhattacharyya \\ SRF, MIU, ISI Kolkata
}




\section{Outline}

- Statistical proximity

- Similarity/dissimilarity measures

- Gene ontology

- Biological proximity

- Dependence analysis

- Conclusions 


\section{Statistical proximity}
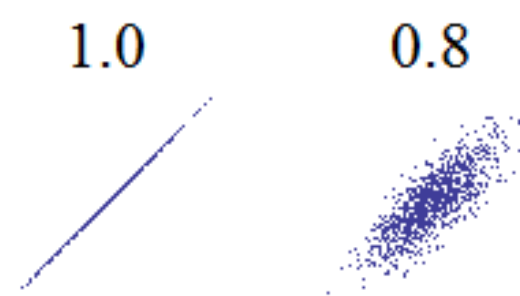

0.4
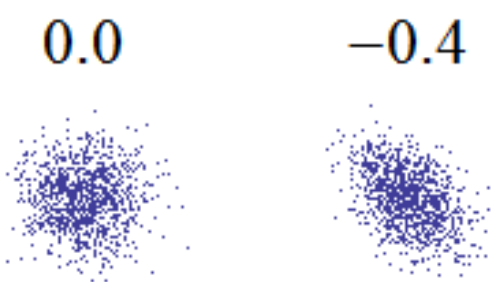

$-0.8$

$-1.0$

1.0

1.0

1.0
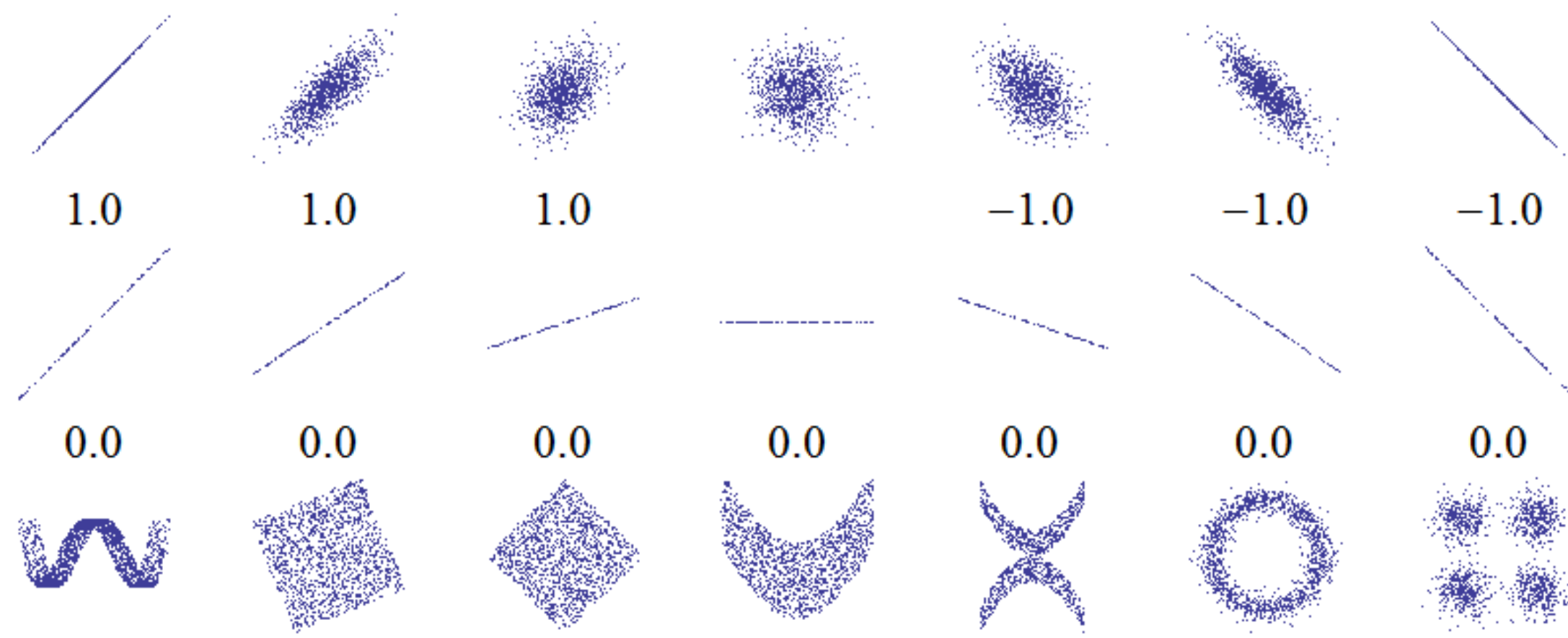


\section{Similarity/dissimilarity measures}

\begin{tabular}{|c|c|c|}
\hline Name & Measure & Type \\
\hline \hline Uncentered correlation coefficient/Cosine & $\left(\mathbf{E}_{i} \bullet \mathbf{E}_{j}\right) /\left(\left\|\mathbf{E}_{i}\right\|\left\|\mathbf{E}_{j}\right\|\right)$ & Similarity \\
\hline Pearson correlation coefficient & $\operatorname{Cov}\left(\mathbf{E}_{i}, \mathbf{E}_{j}\right) /\left(\sigma_{\mathbf{E}_{i}}{ }^{\sigma} \mathbf{E}_{j}\right)$ & Similarity \\
\hline Spearman's rank correlation & $\rho\left(\operatorname{Ranked}\left(\mathbf{E}_{i}\right), \operatorname{Ranked}\left(\mathbf{E}_{j}\right)\right)$ & Similarity \\
\hline Cross-correlation 1 & $\left(\frac{1-\rho\left(\mathbf{E}_{i}, \mathbf{E}_{j}\right)}{1+\rho\left(\mathbf{E}_{i}, \mathbf{E}_{j}\right)}\right)^{\beta}$ & Distance \\
\hline Cross-correlation 2 & $\sqrt{2\left(1-\rho\left(\mathbf{E}_{i}, \mathbf{E}_{j}\right)\right)}$ & Distance \\
\hline Root mean square & $\frac{1}{n} \sqrt{\left\|\mathbf{E}_{i}-\mathbf{E}_{j}\right\|^{2}}$ & Distance \\
\hline Minkowski & $\sqrt[p]{\left.\left\|\mathbf{E}_{i}-\mathbf{E}_{j}\right\|\right|^{p}}$ & Distance \\
\hline Squared Euclidean & || $\mathbf{E}_{i}-\mathbf{E}_{j} \|^{2}$ & Distance \\
\hline City block/Manhattan & $\left|\mathbf{E}_{i}-\mathbf{E}_{j}\right|$ & Distance \\
\hline Chebyshev & $\max _{t}\left(\left|\mathbf{E}_{i}(t)-\mathbf{E}_{j}(t)\right|\right)$ & Distance \\
\hline Kullback-Leibler & $\sum_{t=1}^{n} e_{j}(t) \ln \frac{e_{j}(t)}{e_{i}(t)}$ & Distance \\
\hline
\end{tabular}




\section{Gene ontology}

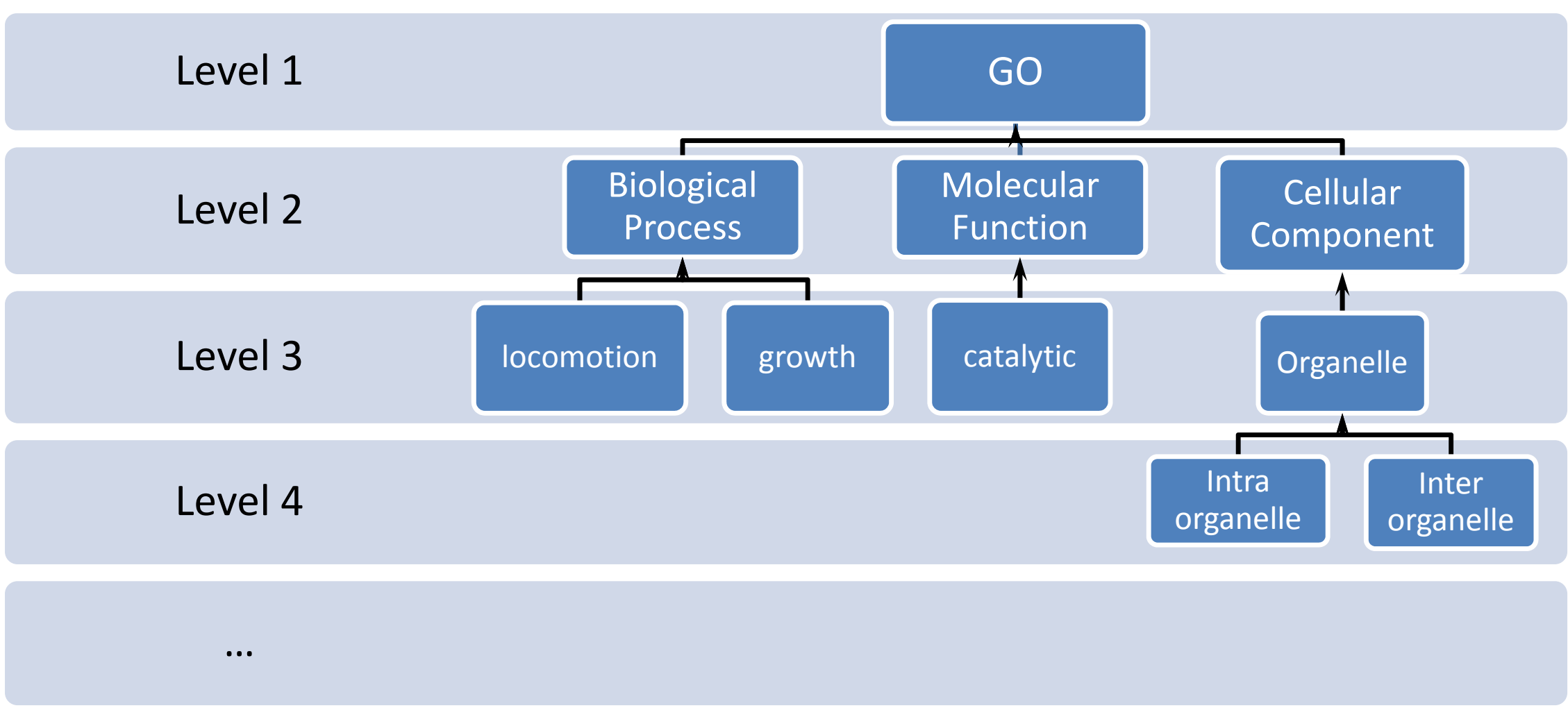

Level $n$ 


\section{Gene ontology (a closer view)}

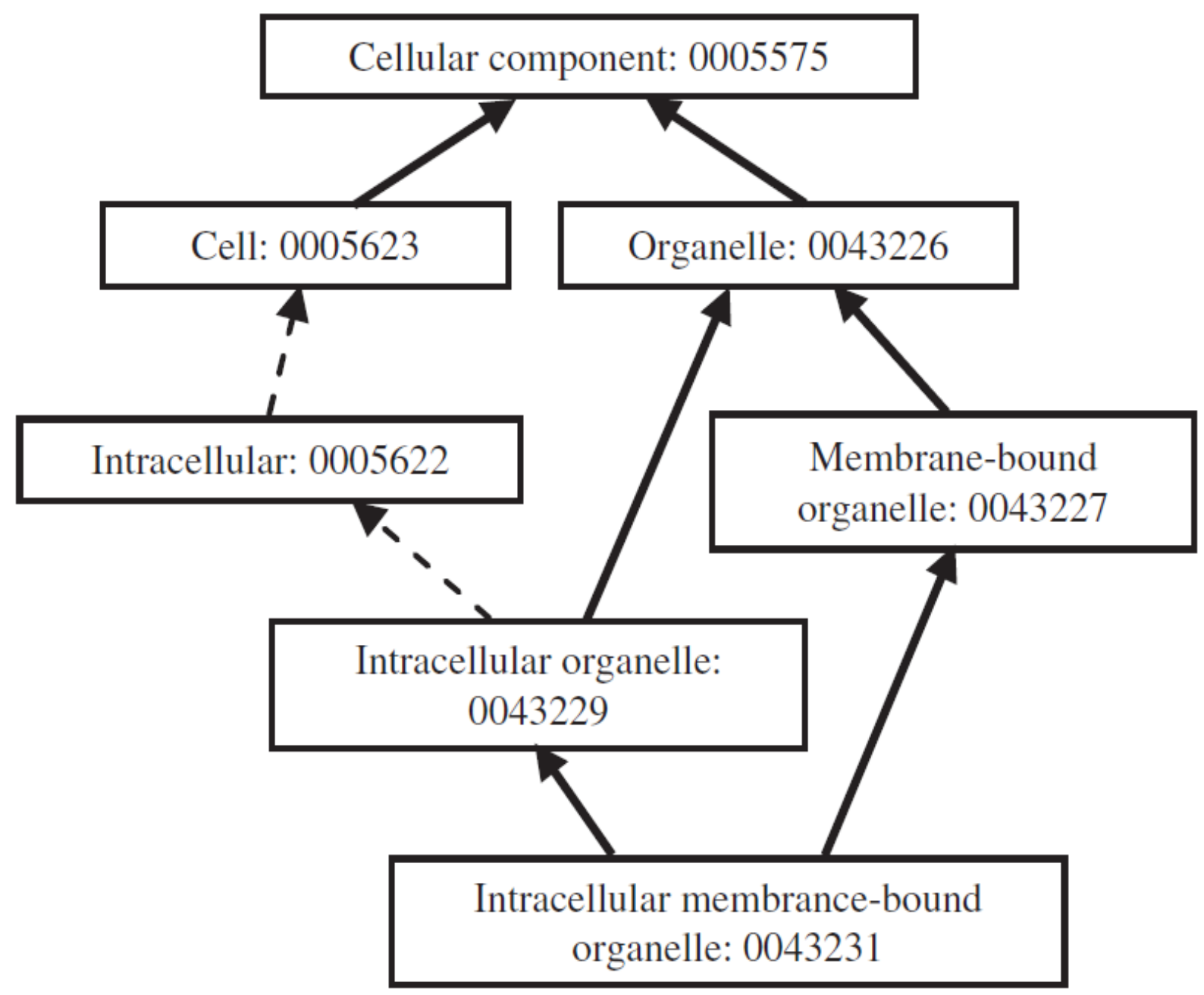




\section{Biological proximity $[1]$}

Let us assume that for a pair of genes $x$ and $y$ the sets of annotated GO terms $T_{x}=\left\{t_{x 1}, t_{x 2}, \ldots, t_{x m}\right\}$ and $T_{y}=$ $\left\{t_{y 1}, t_{y 2}, \ldots, t_{y n}\right\}$ are given, respectively. Then, the seman额ic similarity is computed as

- Jaccard similarity: $S S(x, y)=\frac{|x \cap y|}{|x \cap y|}$

- Dice similarity: $S S(x, y)=\frac{2|x \cap y|}{|x|+|y|}$ 


\section{Dependence analysis}

\begin{tabular}{|c|c|c|c|}
\hline Similarity Measures & BP & CC & MF \\
\hline \hline Cosine & 0.50 & 0.58 & 0.52 \\
\hline Pearson correlation & 0.51 & 0.62 & 0.52 \\
\hline Spearman's rank correlation & $\mathbf{0 . 5 2}$ & 0.59 & $\mathbf{0 . 5 3}$ \\
\hline Cross-correlation 1 & 0.50 & 0.59 & 0.50 \\
\hline Cross-correlation 2 & 0.51 & $\mathbf{0 . 6 3}$ & $\mathbf{0 . 5 3}$ \\
\hline Root mean square & 0.51 & 0.55 & 0.52 \\
\hline Minkowski $(p=3)$ & 0.51 & 0.55 & 0.51 \\
\hline Squared Euclidean & 0.51 & 0.54 & 0.51 \\
\hline Manhattan/City block & 0.51 & 0.55 & 0.52 \\
\hline Chebyshev & 0.51 & 0.55 & 0.51 \\
\hline Kullback-Leibler & 0.51 & 0.55 & $\mathbf{0 . 5 3}$ \\
\hline
\end{tabular}




\section{Dependence analysis (continued)}

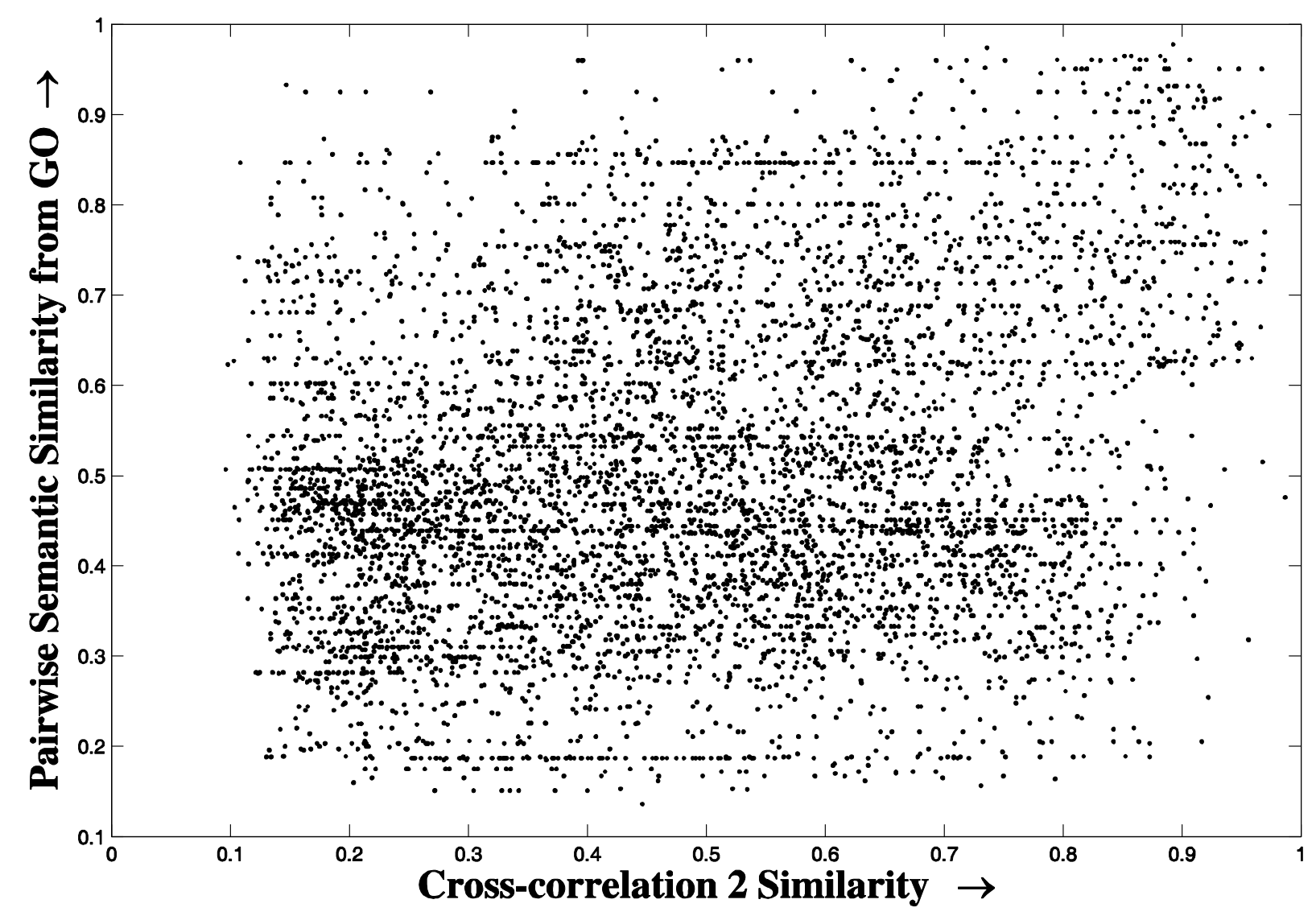




\section{Dependence analysis (continued)}

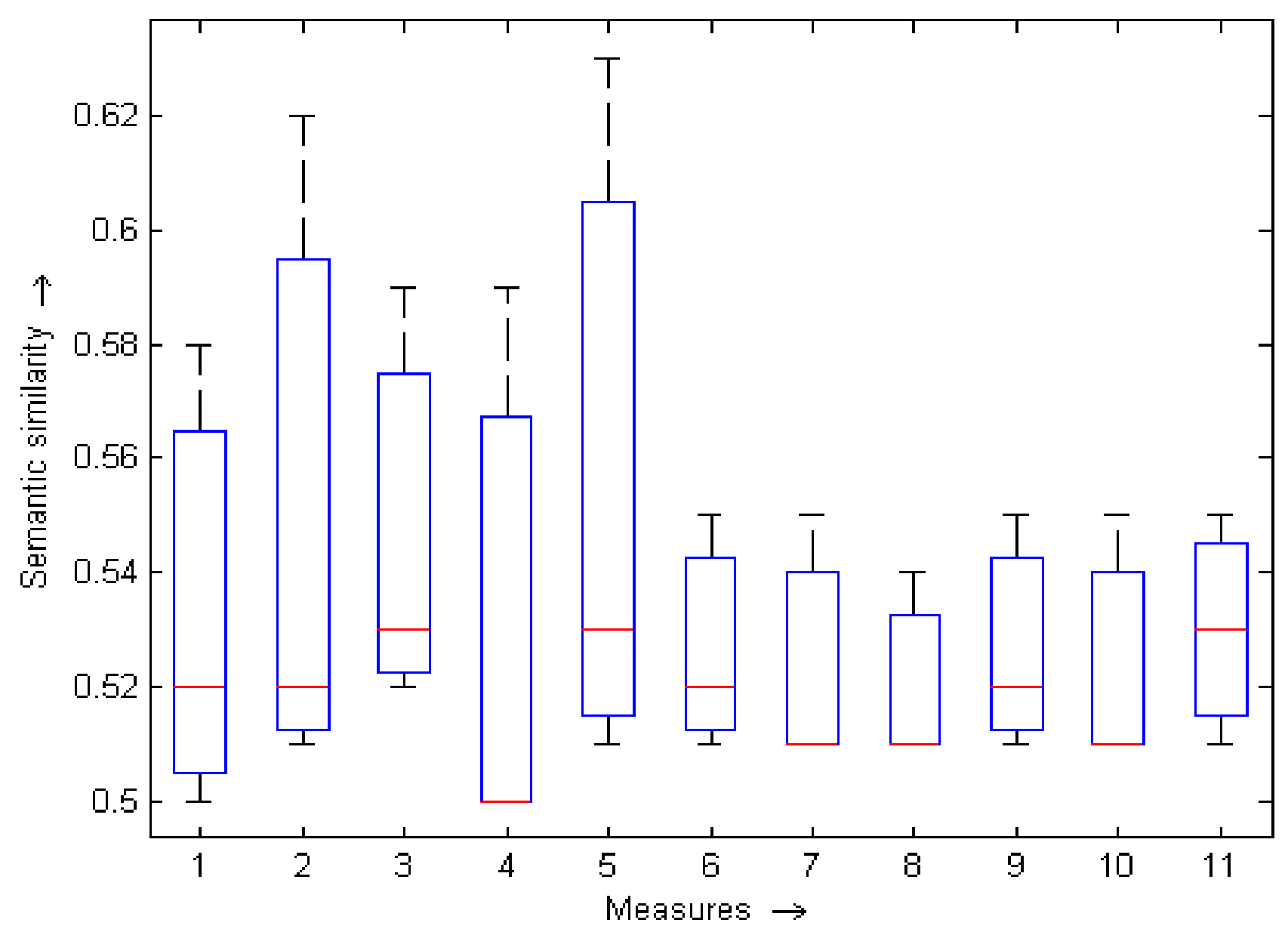




\section{Results on BioSim}

- For BP: 0.53

- For MF: 0.56

- For CC: 0.54 


\section{Biological evaluation of clusters}

$$
\begin{aligned}
& \mathcal{S}_{B P}(G)=\sum_{x, y \in G} S S(x, y) \\
& \mathcal{S}_{M F}(G)=\sum_{x, y \in G} S S(x, y) \\
& \text { and, } \mathcal{S}_{C C}(G)=\sum_{x, y \in G} S S(x, y), \text { respectively. }
\end{aligned}
$$




\section{Conclusions}

- More robust understanding of gene ontology

- More robust measure 


\section{References}

1. P. W. Lord, R. D. Stevens, A. Brass and C. A. Goble, "Investigating semantic similarity measures across the Gene Ontology: the relationship between sequence and annotation," Bioinformatics, 19(10):1275-1283, 2003.

2. J. Z. Wang, Z. Du, R. Payattakool, P. S. Yu and C. -F. Chen, "A new method to measure the semantic similarity of GO terms," Bioinformatics, 23(10):1274-1281, 2007.

3. H. Wang, F. Azuaje, O. Bodenreider and J. Dopazo, "Gene Expression Correlation and Gene Ontology-Based Similarity: An Assessment of Quantitative Relationships," In Proceedings of the IEEE Symposium on Computational Intelligence in Bioinformatics and Computational Biology, pp. 25-31, 2004.

4. M. Popescu, J. M. Keller, and J. A. Mitchell, "Fuzzy measures on the gene ontology for gene product similarity," IEEE/ACM Transactions on Computational Biology and Bioinformatics, 3(3):263-274, 2006.

5. M. Mistry and P. Pavlidis, "Gene ontology term overlap as a measure of gene functional similarity," BMC Bioinformatics, 9:327, 2008.

6. S. Bandyopadhyay and M. Bhattacharyya, "," IEEE/ACM Transactions on Computational Biology and Bioinformatics, 2010 (in press). 
Thank you 